Discrete Comput Geom 26:307-320 (2001)

DOI: $10.1007 / \mathrm{s} 00454-001-0039-6$

\title{
Exact and Approximation Algorithms for Minimum-Width Cylindrical Shells*
}

\author{
P. K. Agarwal, ${ }^{1}$ B. Aronov, ${ }^{2}$ and M. Sharir ${ }^{3}$ \\ ${ }^{1}$ Center for Geometric Computing, Department of Computer Science, \\ Box 90129, Duke University, \\ Durham, NC 27708-0129, USA \\ pankaj@cs.duke.edu \\ ${ }^{2}$ Department of Computer and Information Science, Polytechnic University, \\ Brooklyn, NY 11201-3840, USA \\ aronov@ziggy.poly.edu \\ ${ }^{3}$ School of Mathematical Sciences, Tel Aviv University, \\ Tel Aviv 69978, Israel \\ sharir@math.tau.ac.il \\ and \\ Courant Institute of Mathematical Sciences, New York University, \\ New York, NY 10012, USA
}

\begin{abstract}
Let $S$ be a set of $n$ points in $\mathbb{R}^{3}$. Let $\omega^{*}$ be the width (i.e., thickness) of a minimum-width infinite cylindrical shell (the region between two co-axial cylinders) containing $S$. We first present an $O\left(n^{5}\right)$-time algorithm for computing $\omega^{*}$, which as far as we know is the first nontrivial algorithm for this problem. We then present an $O\left(n^{2+\delta}\right)$ time algorithm, for any $\delta>0$, that computes a cylindrical shell of width at most $56 \omega^{*}$ containing $S$.
\end{abstract}

* Work by P.A. was supported by Army Research Office MURI Grant DAAH04-96-1-0013, by a Sloan fellowship, by NSF Grants EIA-9870724, and CCR-9732787, by an NYI award, and by a grant from the U.S.Israeli Binational Science Foundation. Work by B.A. was supported by a Sloan Research Fellowship, by NSF Grant CCR-99-72568, and by a grant from the U.S.-Israeli Binational Science Foundation. Work by M.S. was supported by NSF Grants CCR-97-32101, CCR-94-24398, by grants from the U.S.-Israeli Binational Science Foundation, the G.I.F., the German-Israeli Foundation for Scientific Research and Development, and the ESPRIT IV LTR Project No. 21957 (CGAL), and by the Hermann Minkowski-MINERVA Center for Geometry at Tel Aviv University. Part of the work by P.A. and B.A. on the paper was done when they visited Tel Aviv University in May 1999. 


\section{Introduction}

Given a line $\ell$ in $\mathbb{R}^{3}$ and two real numbers $0 \leq r \leq R$, the cylindrical shell $\Sigma(\ell, r, R)$ is the closed region lying between the two co-axial cylinders of radii $r$ and $R$ with $\ell$ as their axis, i.e.,

$$
\Sigma(\ell, r, R)=\left\{p \in \mathbb{R}^{3} \mid r \leq d(p, \ell) \leq R\right\}
$$

where $d(p, \ell)$ is the Euclidean distance between point $p$ and line $\ell$. The width of $\Sigma(\ell, r, R)$ is $R-r$. Let $S$ be a set of $n$ points in $\mathbb{R}^{3}$. One can measure how well $S$ fits a cylindrical surface by computing a cylindrical surface $\mathcal{C}=\mathcal{C}(S)$ so that the maximum distance between any point of $S$ and $\mathcal{C}$ is minimized. If $\ell$ and $\rho$ are the axis and the radius of $\mathcal{C}$ and $\theta$ is the maximum distance between $\mathcal{C}$ and $S$, then $S \subset \Sigma(\ell, \rho-\theta, \rho+\theta)$. Hence, the problem of approximating $S$ by a cylindrical surface is equivalent to computing a cylindrical shell $\Sigma^{*}(S)$ of minimum width that contains $S$.

The main motivation for computing a minimum-width cylindrical shell comes from computational metrology. In order to measure the quality of a manufactured cylinder $\Gamma$, we sample a set $S$ of points on the surface of $\Gamma$ using coordinate measuring machines and then fit a cylindrical surface through $S$ so that the maximum distance between the points of $S$ and the cylinder is minimized. For example, this is one of the criteria suggested in the recent ASME Y14.5M standard to determine how closely $\Gamma$ resembles a cylinder [17], [18].

In the last few years much work has been done on measuring the circularity of a planar point set, which is defined as the width of the thinnest annulus that contains the point set [2], [5], [11]-[14]. The best known exact algorithm runs in $O\left(n^{3 / 2+\delta}\right)$ time, for any $\delta>0$ [5], and near-linear approximation algorithms are proposed in [2], [9], and [11]. In three dimensions, Chan [9] has shown that the minimum-width spherical shell (a region enclosed between two concentric spheres) containing an $n$-element point set $S$ can be computed in time $O\left(n^{2}\right)$. The same paper also presents linear-time algorithms that compute an approximation to the minimum-width enclosing spherical shell in any dimension; see also [2]. There has also been some work on computing the smallest cylinder enclosing a point set in $\mathbb{R}^{3}$ [1], [15]. Agarwal et al. [1] developed an $O\left(n^{3+\delta}\right)$ time algorithm, for any $\delta>0$, for computing the smallest enclosing cylinder. They also proposed a $(1+\varepsilon)$-approximation algorithm (i.e., an algorithm that produces an enclosing cylinder whose radius is at most $(1+\varepsilon)$ times the minimum radius) that runs in $O\left(n / \varepsilon^{2}\right)$ time. This has been improved by Chan [9] to $O(n / \varepsilon)$ or to $O(n+$ $\left.1 / \varepsilon^{3}\right)$.

Finding the minimum-width cylindrical shell $\Sigma^{*}(S)$ that contains a given point set is harder than computing a minimum-width enclosing spherical shell, computing a smallest enclosing cylinder, or computing a thinnest annulus containing a planar point set. Actually, the second and third problems are special cases of computing a thinnest cylindrical shell-finding a smallest enclosing cylinder is the same as finding a minimum-width cylindrical shell whose inner radius is 0 ; and finding a thinnest cylindrical shell with the axis parallel to a given direction $\mathbf{n}$ is the same as finding a thinnest annulus containing the projection of $S$ in direction $\mathbf{n}$ onto a plane orthogonal to $\mathbf{n}$. Since a cylindrical shell is specified by six parameters-four parameters define the axis of the shell and the remaining two define the inner and outer radii of the shell $-\Sigma^{*}(S)$ is in general "defined" 
by a subset $A \subset S$ of six points, in the sense that $\Sigma^{*}(S)$ is one of the $O(1)$ cylindrical shells that contain $A$ on their inner and outer boundaries. This suggests the following naïve procedure for computing $\Sigma^{*}(S)$ : For each subset $A \subseteq S$ of size six, compute the $O(1)$ cylindrical shells containing $A$ on their inner and outer boundary. For each such shell $\Sigma$, check in $O(n)$ time whether $S \subset \Sigma$. Return the thinnest among those shells that contain $S$. This naïve approach leads to an $O\left(n^{7}\right)$-algorithm for computing $\Sigma^{*}(S)$ under an appropriate model of computation in which the roots of a fixed-degree polynomial can be computed in $O(1)$ time. As the first result of this paper, we describe, in Section 2, an improved $O\left(n^{5}\right)$-time algorithm for computing $\Sigma^{*}(S)$. We are not aware of any faster algorithm for the exact problem. Recently, Devillers and Preparata proposed a linear-time constant-factor approximation algorithm for the minimum-width cylindrical shell problem under the assumption that the points are "almost" cylindrical [10].

Since computing $\Sigma^{*}(S)$ is so expensive, we develop a more efficient approximation algorithm for computing a cylindrical shell that contains $S$ and has width at most $c \omega^{*}$, where $\omega^{*}$ is the width of $\Sigma^{*}(S)$ and $c$ is a constant. We first prove in Section 3 a Helly-type theorem for $\Sigma^{*}(S)$, which we believe to be of independent interest, and which asserts roughly the following: Let $A \subseteq S$ be a subset of four points so that the volume of the tetrahedron spanned by $A$ is close to the largest volume of a tetrahedron spanned by any four points of $S$. For a direction $\mathbf{n} \in \mathbb{S}^{2}$ and a point set $X$, let $\omega^{*}(X, \mathbf{n})$ denote the minimum width of a cylindrical shell containing $X$ and with axis direction $\mathbf{n}$. Then for any direction $\mathbf{n}, \omega^{*}(S, \mathbf{n}) \leq c \cdot \max _{p \in S} \omega^{*}(A \cup\{p\}, \mathbf{n})$, for an absolute constant $c>1$. The constant that our analysis yields is about 56 , but we believe that the theorem also holds with a much smaller constant. Using this observation, we develop in Section 4 an $O\left(n^{2+\delta}\right)$-time algorithm, for any $\delta>0$, to compute a cylindrical shell of width at most about $56 \omega^{*}$ that contains $S$.

\section{Computing $\Sigma^{*}(S)$ Exactly}

In this section we describe an $O\left(n^{5}\right)$-time algorithm for computing $\Sigma^{*}(S)$. Without loss of generality assume that the axis of $\Sigma^{*}(S)$ is not parallel to the $x y$-plane; the case of a horizontal axis can be handled by a simpler algorithm, whose details are omitted. A cylinder $C$ with a nonhorizontal axis $a$ can be parametrized by a 5 -tuple $\left(a_{1}, a_{2}, a_{3}, a_{4}, r\right)$, where $r$ is the radius of $C$ and where the axis of $C$ is the line $a=\{p+t q \mid t \in \mathbb{R}\}$, $p=\left(a_{1}, a_{2}, 0\right)$ is the intersection point of $a$ with the $x y$-plane, and $q=\left(a_{3}, a_{4}, 1\right)$ is the direction vector of $a$. Let $x$ be a point in $\mathbb{R}^{3}$. The orthogonal projection of $x$ to the line $a$ is $p+((x-p) \cdot q /\|q\|) q /\|q\|=p+\left((x-p) \cdot q /\|q\|^{2}\right) q$. Hence, the distance between $x$ and $a$ is

$$
d(x, a)=\left\|(p-x)-\frac{(p-x) \cdot q}{\|q\|^{2}} q\right\| .
$$

Since $x$ lies in the cylinder $C$ if and only if $d(x, a) \leq r$, after some algebraic manipulation we obtain that $x=\left(x_{1}, x_{2}, x_{3}\right)$ lies inside $C$ if and only if

$$
f\left(x_{1}, x_{2}, x_{3}, a_{1}, a_{2}, a_{3}, a_{4}\right) \leq\left(a_{3}^{2}+a_{4}^{2}+1\right) r^{2},
$$


where

$$
\begin{aligned}
f\left(x_{1}, x_{2}, x_{3},\right. & \left.a_{1}, a_{2}, a_{3}, a_{4}\right) \\
= & {\left[\left(a_{4}^{2}+1\right) a_{1}^{2}+\left(a_{3}^{2}+1\right) a_{2}^{2}-2 a_{1} a_{2} a_{3} a_{4}\right]+2\left[a_{2} a_{3} a_{4}-a_{1}\left(a_{4}^{2}+1\right)\right] x_{1} } \\
& +2\left[a_{1} a_{3} a_{4}-a_{2}\left(a_{3}^{2}+1\right)\right] x_{2}+2\left[a_{1} a_{3}+a_{2} a_{4}\right] x_{3}-2\left[a_{3} a_{4}\right] x_{1} x_{2} \\
& -2\left[a_{3}\right] x_{1} x_{3}-2\left[a_{4}\right] x_{2} x_{3}+[1]\left(x_{1}^{2}+x_{2}^{2}\right)+\left[a_{3}^{2}\right]\left(x_{2}^{2}+x_{3}^{2}\right) \\
& +\left[a_{4}^{2}\right]\left(x_{1}^{2}+x_{3}^{2}\right) .
\end{aligned}
$$

Hence, a point $x$ lies in a cylindrical shell $\sigma=\left(a_{1}, a_{2}, a_{3}, a_{4}, r, R\right)$ with axis $a=$ $\left(a_{1}, a_{2}, a_{3}, a_{4}\right)$, parametrized as above, inner radius $r$, and outer radius $R$ if and only if

$$
r^{2}\left(a_{3}^{2}+a_{4}^{2}+1\right) \leq f\left(x_{1}, x_{2}, x_{3}, a_{1}, a_{2}, a_{3}, a_{4}\right) \leq R^{2}\left(a_{3}^{2}+a_{4}^{2}+1\right) .
$$

We set

$$
\begin{aligned}
\varphi_{1}(\sigma) & =a_{2} a_{3} a_{4}-a_{1}\left(a_{4}^{2}+1\right), \\
\varphi_{2}(\sigma) & =a_{1} a_{3} a_{4}-a_{2}\left(a_{3}^{2}+1\right), \\
\varphi_{3}(\sigma) & =a_{1} a_{3}+a_{2} a_{4}, \\
\varphi_{4}(\sigma) & =a_{3} a_{4}, \\
\varphi_{5}(\sigma) & =a_{3}, \\
\varphi_{6}(\sigma) & =a_{4}, \\
\varphi_{7}(\sigma) & =a_{3}^{2}, \\
\varphi_{8}(\sigma) & =a_{4}^{2}, \\
\varphi_{9}(\sigma) & =r^{2}\left(a_{3}^{2}+a_{4}^{2}+1\right)-\left(a_{4}^{2}+1\right) a_{1}^{2}-\left(a_{3}^{2}+1\right) a_{2}^{2}+2 a_{1} a_{2} a_{3} a_{4}, \\
\varphi_{10}(\sigma) & =R^{2}\left(a_{3}^{2}+a_{4}^{2}+1\right)-\left(a_{4}^{2}+1\right) a_{1}^{2}-\left(a_{3}^{2}+1\right) a_{2}^{2}+2 a_{1} a_{2} a_{3} a_{4}, \\
\psi_{0}(x) & =x_{1}^{2}+x_{2}^{2}, \quad \psi_{1}(x)=2 x_{1}, \\
\psi_{2}(x) & =2 x_{2}, \\
\psi_{4}(x) & =-2 x_{1} x_{2}, \quad \psi_{5}(x)=-2 x_{1} x_{3}, \\
\psi_{6}(x) & =-2 x_{2} x_{3}, \quad \psi_{7}(x)=x_{2}^{2}+x_{3}^{2}, \\
\psi_{8}(x) & =x_{1}^{2}+x_{3}^{2} .
\end{aligned}
$$

Then the constraint (2.2) can be rewritten as a linear constraint:

$$
H_{x}(\sigma): \varphi_{9}(\sigma) \leq \psi_{0}(x)+\sum_{i=1}^{8} \varphi_{i}(\sigma) \psi_{i}(x) \leq \varphi_{10}(\sigma) .
$$

For any point $p \in \mathbb{R}^{3}$, define the wedge $H_{p} \subset \mathbb{R}^{10}$, formed by the intersection of two halfspaces, as

$$
H_{p}=\left\{\left(y_{1}, \ldots, y_{10}\right) \mid y_{9} \leq \psi_{0}(p)+\sum_{i=1}^{8} y_{i} \psi_{i}(p) \leq y_{10}\right\} .
$$


Set $\varphi(\sigma)=\left\langle\varphi_{1}(\sigma), \ldots, \varphi_{10}(\sigma)\right\rangle \in \mathbb{R}^{10}$. Let $P=\bigcap_{p \in S} H_{p}$ be the convex polyhedron defined by the intersection of the $2 n$ corresponding halfspaces. $P$ has $O\left(n^{5}\right)$ faces and can be computed in $O\left(n^{5}\right)$ time [8]. A cylindrical shell (with nonhorizontal axis) $\sigma$ contains $S$ if and only if $\varphi(\sigma) \in P$.

Let $\Psi \subseteq \mathbb{R}^{4} \times\left(\mathbb{R}^{+}\right)^{2}$ denote the six-dimensional set of all cylindrical shells (with nonhorizontal axis) that contain $S$. Then $\varphi(\Psi)$ is the intersection of $P$ with the sixdimensional surface $\Phi=\left\{\varphi(\sigma) \mid \sigma \in \mathbb{R}^{4} \times\left(\mathbb{R}^{+}\right)^{2}\right\}$. After having computed $P, \Psi$ can be computed in $O\left(n^{5}\right)$ time, e.g., by triangulating $P$ into $O\left(n^{5}\right)$ simplices and then, for every simplex $\tau$ in the triangulation, computing $\tau \cap \Phi$. Finally, for each simplex $\tau$, we compute in $O$ (1) time (under an appropriate model of computation in which the roots of a constantdegree polynomial can be computed in $O(1)$ time) the minimum-width cylindrical shell $\sigma$ such that $\varphi(\sigma) \in \tau \cap \varphi(\Psi)$. Hence, we have established the following result.

Theorem 2.1. Given a set $S$ of $n$ points in $\mathbb{R}^{3}$, a minimum-width cylindrical shell containing $S$ can be computed in $O\left(n^{5}\right)$ time.

\section{A Helly-Like Property of Cylindrical Shells}

Let $S$ be a set of $n$ points in $\mathbb{R}^{3}$, and let $t>1$ be a constant. For any finite point set $X \subset \mathbb{R}^{3}$ of at least four points, let $\mu(X)$ denote the maximum volume of a simplex spanned by four points of $X$. Let $T$ be a tetrahedron spanned by points of $S$ whose volume is $\mu(S) / t$. Let $A=\left\{a_{1}, \ldots, a_{4}\right\} \subseteq S$ denote the set of vertices of $T$. The simplex $T$ has the following useful property.

Lemma 3.1. Let $f$ be any $k$-flat, for $k=0,1,2$. Then for any $p \in S$ we have

$$
d(p, f) \leq(4 t-1) \cdot \max _{1 \leq i \leq 4} d\left(a_{i}, f\right) .
$$

Proof. Let $\Delta \subset \mathbb{R}^{3}$ be the locus of all points $q$ so that each of the simplices $a_{1} a_{2} a_{3} q$, $a_{1} a_{2} a_{4} q, a_{1} a_{3} a_{4} q$, and $a_{2} a_{3} a_{4} q$ has volume at most $t \cdot \operatorname{Vol}(T)$; see Fig. 1. By assumption,

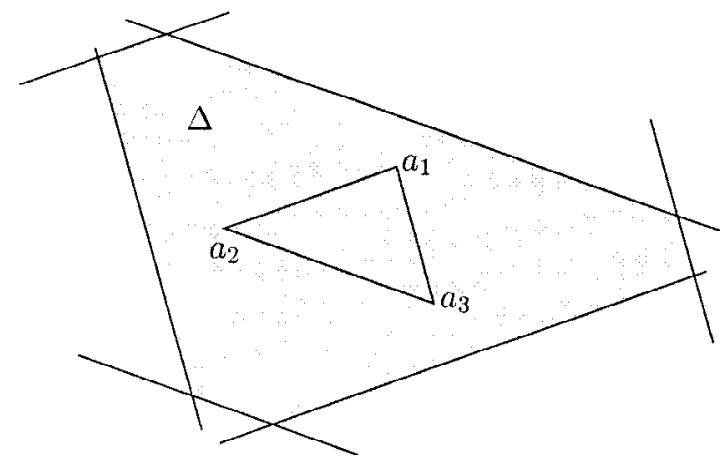

Fig. 1. A two-dimensional version of the region $\Delta$, for $t$ slightly larger than 1 . 
we have $S \subset \Delta$. Let $h_{i}$ be the plane containing $A \backslash\left\{a_{i}\right\}$, and let $\Lambda_{i}$ be the slab bounded by two planes parallel to $h_{i}$ and at distance $t \cdot d\left(a_{i}, h_{i}\right)$ from it. Then $\Delta=\bigcap_{i=1}^{4} \Lambda_{i}$; see Fig. 1 . Using barycentric coordinates, we can represent any point $q \in \Delta$ as $q=\sum_{i=1}^{4} \lambda_{i} a_{i}$, where $\sum_{i=1}^{4} \lambda_{i}=1$ and $\left|\lambda_{i}\right| \leq t$, for $i=1, \ldots, 4$. For $i=1, \ldots, 4$, let $b_{i}$ be the point in $f$ nearest to $a_{i}$, and put $q^{*}=\sum_{i=1}^{4} \lambda_{i} b_{i} \in f$. We then have

$$
\begin{aligned}
d(q, f) & \leq d\left(q, q^{*}\right) \\
& =d\left(\sum_{i=1}^{4} \lambda_{i} a_{i}, \sum_{i=1}^{4} \lambda_{i} b_{i}\right) \\
& =\left\|\sum_{i=1}^{4} \lambda_{i}\left(a_{i}-b_{i}\right)\right\| \\
& \leq \sum_{i=1}^{4}\left|\lambda_{i}\right| d\left(a_{i}, f\right) \\
& \leq(4 t-1) \cdot \max _{1 \leq i \leq 4} d\left(a_{i}, f\right),
\end{aligned}
$$

for each $q \in \Delta$, where the last inequality follows by observing that $\max \sum_{i=1}^{4}\left|\lambda_{i}\right|$, subject to $\sum_{i=1}^{4} \lambda_{i}=1$ and $\left|\lambda_{i}\right| \leq t$ for $i=1, \ldots, 4$, is $4 t-1$. This implies the assertion of the lemma.

Fix a direction $\mathbf{n} \in \mathbb{S}^{2}$, the unit sphere of directions, and let $\pi=\pi^{(\mathbf{n})}$ be the plane normal to $\mathbf{n}$ and passing through the origin. For a point $x \in \mathbb{R}^{3}$, let $x^{*}$ denote its orthogonal projection to $\pi$. Set $S^{*}=\left\{p^{*} \mid p \in S\right\}$. Similarly, define $A^{*}$ to be the projection of $A$ to $\pi$.

\section{Corollary 3.2.}

(i) Let $o$ and $\rho$ be the center and radius of the smallest disk enclosing $A^{*}$. Then $S^{*}$ is contained in the disk of radius $(4 t-1) \rho$ centered at $o$.

(ii) For any line $\ell$ lying in $\pi$,

$$
\max _{p \in S} d\left(p^{*}, \ell\right) \leq(4 t-1) \max _{a \in A} d\left(a^{*}, \ell\right) .
$$

Proof. Part (i) follows by applying Lemma 3.1 to the line in direction $\mathbf{n}$ and passing through $o$. The second part is proved by applying Lemma 3.1 to the plane orthogonal to $\pi$ and passing through $\ell$.

The following geometric lemma lies at the heart of the main result of this section. Let $D(x, \delta)$ denote the disk of diameter $\delta$ centered at a point $x$.

Lemma 3.3. Let $\triangle a b c$ be a triangle in the plane, and let $\tau \geq 1$ and $0<\omega<$ Width $(\triangle a b c) / 3.4$ be two parameters. Define $\Delta=\Delta(\tau)$ to be the locus of all points $x$ such that the area of each of the triangles $\triangle a b x, \triangle a c x, \Delta b c x$ is at most $\tau$ times the 


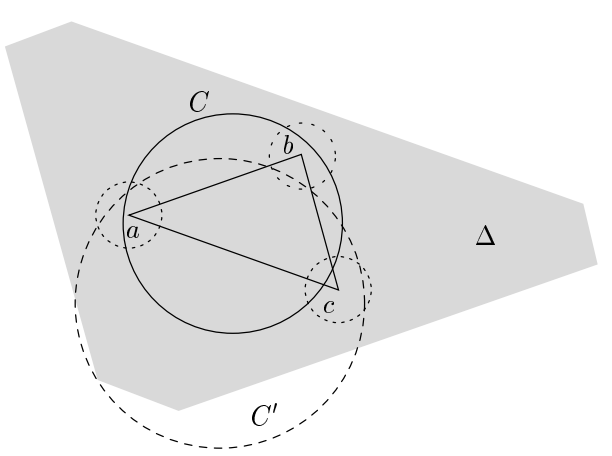

(i)

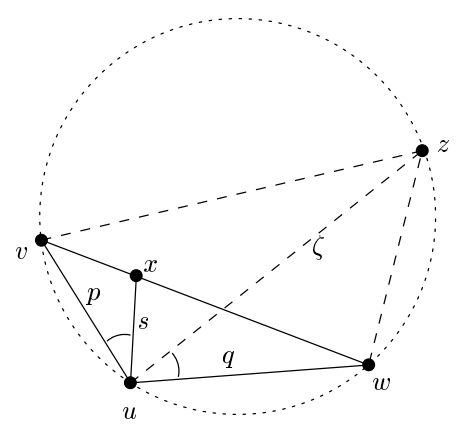

(ii)

Fig. 2. (i) Setup of the lemma; (ii) geometric interpretation of the inversion.

area of $\triangle a b c$. Let $C$ and $C^{\prime}$ be two circles, each of which meets all three disks $D(a, \omega)$, $D(b, \omega), D(c, \omega)$. Then for any $z \in C \cap \Delta$ we have

$$
d\left(z, C^{\prime}\right) \leq(6.95 \tau+3.5) \omega
$$

(see Fig. 2(i)).

Remark 3.4. Informally, the lemma asserts that if two circles are close to each other near the three points $a, b, c$, then they remain close to each other within $\Delta$. Without confinement to $\Delta$, the assertion may fail, as is easily checked.

Proof. We parametrize points on $C$ using inversion, as follows. Pick points $u \in C \cap$ $D(a, \omega), v \in C \cap D(b, \omega), w \in C \cap D(c, \omega)$. (Note that the condition on $\omega$ implies that the disks $D(a, \omega), D(b, \omega), D(c, \omega)$ are pairwise disjoint.) Without loss of generality, we may assume that the order of $u, v, w$, and $z$ along $C$ in the clockwise direction is $u, v, z, w$. Write $v=u+p, w=u+q$, and $z=u+\zeta$. Apply an inversion to the plane that takes $u$ to infinity. For example, using complex numbers, we may use the transformation $\xi \mapsto 1 /(\xi-u)$. This transformation maps $C$ to a straight line containing the images $1 / p, 1 / q$, and $1 / \zeta$ of $v, w$, and $z$, respectively, so that $1 / \zeta$ lies between $1 / p$ and $1 / q$. Hence there is a real parameter $\lambda \in[0,1]$, such that

$$
\frac{1}{\zeta}=\frac{\lambda}{p}+\frac{1-\lambda}{q}
$$

or

$$
\zeta=\frac{p q}{\lambda q+(1-\lambda) p} .
$$

The following geometric interpretation will be useful in the subsequent analysis. Put $s=\lambda q+(1-\lambda) p$ and $x=u+s$. The point $x$ lies on the edge $v w$ of the triangle $u v w$ and splits it in the ratio $\lambda:(1-\lambda)$; that is $|x-v|=\lambda|w-v|$ and $|x-w|=(1-\lambda)|w-v|$. Since $p q=\zeta s$ (or $p / s=\zeta / q$ ), the triangles $\Delta v u x$ and $\triangle z u w$ are similar. Analogously, $\triangle w u x$ and $\Delta z u v$ are similar. See Fig. 2(ii). 
This implies that

$$
\frac{\lambda|w-v|}{|s|}=\frac{|w-z|}{|q|} \text { and } \frac{(1-\lambda)|w-v|}{|s|}=\frac{|v-z|}{|p|} \text {. }
$$

Since $u, v, z, w$ are cocircular, $\measuredangle v u w=\pi-\measuredangle v z w$, therefore $\sin (\measuredangle v u w)=\sin (\measuredangle v z w)$. Multiplying the two equalities in (3.3), we obtain

$$
\begin{aligned}
\lambda(1-\lambda)|w-v|^{2} & =|s|^{2} \cdot \frac{|v-z||w-z|}{|p||q|} \\
& =|s|^{2} \cdot \frac{|v-z| \cdot|w-z| \sin (\measuredangle v z w)}{|p| \cdot|q| \sin (\measuredangle v u w)} \\
& =|s|^{2} \cdot \frac{\operatorname{Area}(\triangle v w z)}{\operatorname{Area}(\triangle u v w)} .
\end{aligned}
$$

We prove below in Corollary 3.6 that

$$
\operatorname{Area}(\triangle v w z) \leq 4.05 \tau \cdot \operatorname{Area}(\triangle u v w) .
$$

Intuitively, this is to be expected because the area of $\Delta u v w$ (resp. $\Delta v w z$ ) is a good approximation of the area of $\triangle a b c$ (resp. $\triangle b c z$ ); a rigorous proof is given in Lemma 3.5 below.

We thus have

$$
\lambda(1-\lambda)|w-v|^{2} \leq 4.05 \tau|s|^{2} .
$$

Let $\theta=\angle u v w$. Using the law of cosines, we have

$$
|s|^{2}=|p|^{2}+\lambda^{2}|w-v|^{2}-2 \lambda|p||w-v| \cos \theta
$$

and

$$
|q|^{2}=|p|^{2}+|w-v|^{2}-2|p||w-v| \cos \theta .
$$

Eliminating $\cos \theta$ from the last two equations, we obtain

$$
|s|^{2}=\lambda|q|^{2}+(1-\lambda)|p|^{2}-\lambda(1-\lambda)|w-v|^{2} .
$$

Combining (3.5) and (3.6), we get

$$
\lambda|q|^{2}+(1-\lambda)|p|^{2} \leq(4.05 \tau+1)|s|^{2} .
$$

Apply a symmetric transformation to parametrize $C^{\prime}$ : Pick points $u^{\prime} \in C^{\prime} \cap D(a, \omega)$, $v^{\prime} \in C^{\prime} \cap D(b, \omega), w^{\prime} \in C^{\prime} \cap D(c, \omega)$. Write $v^{\prime}=u^{\prime}+p^{\prime}, w^{\prime}=u^{\prime}+q^{\prime}$, and put

$$
z^{\prime}=u^{\prime}+\frac{p^{\prime} q^{\prime}}{\lambda q^{\prime}+(1-\lambda) p^{\prime}} \in C^{\prime}
$$

Set

$$
\delta=\frac{p q}{\lambda q+(1-\lambda) p}-\frac{p^{\prime} q^{\prime}}{\lambda q^{\prime}+(1-\lambda) p^{\prime}} .
$$


Put $\xi=p^{\prime}-p$ and $\eta=q^{\prime}-q$. Observe that $|\xi|,|\eta| \leq \omega$. We have

$$
\begin{aligned}
|\delta| & =\left|\frac{p q}{\lambda q+(1-\lambda) p}-\frac{(p+\xi)(q+\eta)}{\lambda(q+\eta)+(1-\lambda)(p+\xi)}\right| \\
& \leq \frac{|\lambda q+(1-\lambda) p| \cdot|\xi| \cdot|\eta|+\lambda|q|^{2}|\xi|+(1-\lambda)|p|^{2}|\eta|}{|\lambda q+(1-\lambda) p| \cdot|\lambda(q+\eta)+(1-\lambda)(p+\xi)|} .
\end{aligned}
$$

The denominator in the last expression is at least $|s|(|s|-\omega)$. Moreover, $|s|$ is larger than the height to $v w$ in $\triangle u v w$. As we show below in Lemma 3.5, this height is at least Width $(\triangle a b c)-\omega \geq 2.4 \omega$ (again, this holds because $\triangle u v w$ is a good approximation of $\triangle a b c)$. Therefore

$$
|\delta| \leq \frac{|s| \omega^{2}+\omega\left(\lambda|q|^{2}+(1-\lambda)|p|^{2}\right)}{|s|(|s|-\omega)}
$$

Using (3.7) and the fact that $|s| \geq 2.4 \omega$, we obtain

$$
\begin{aligned}
|\delta| & \leq\left(\frac{1}{|s| / \omega-1}+\frac{4.05 \tau+1}{1-\omega /|s|}\right) \omega \\
& \leq\left(\frac{5}{7}+\frac{12(4.05 \tau+1)}{7}\right) \omega \\
& <(6.95 \tau+2.5) \omega .
\end{aligned}
$$

Therefore,

$$
\begin{aligned}
d\left(z, C^{\prime}\right) & \leq d\left(z, z^{\prime}\right) \leq d\left(u, u^{\prime}\right)+|\delta| \\
& \leq(6.95 \tau+3.5) \omega .
\end{aligned}
$$

This completes the proof of the lemma.

We still need to establish the following lemma.

\section{Lemma 3.5.}

(a) $\operatorname{Area}(\triangle u v w) \geq \frac{94}{289} \operatorname{Area}(\triangle a b c)$.

(b) $\operatorname{Area}(\triangle v w z) \leq\left(\frac{22}{17} \tau+\frac{25}{1156}\right) \operatorname{Area}(\triangle a b c)$.

(c) $|\operatorname{Width}(\triangle u v w)-\operatorname{Width}(\triangle a b c)| \leq \omega$.

Proof. We have

$$
2 \operatorname{Area}(\triangle a b c)=|\overrightarrow{a b} \times \overrightarrow{a c}|
$$

and

$$
2 \operatorname{Area}(\Delta u v w)=|\overrightarrow{u v} \times \overrightarrow{u w}|=|(\overrightarrow{a b}+\overrightarrow{u a}+\overrightarrow{b v}) \times(\overrightarrow{a c}+\overrightarrow{u a}+\overrightarrow{c w})| .
$$

Put $\vec{p}=\overrightarrow{u a}+\overrightarrow{b v}$ and $\vec{q}=\overrightarrow{u a}+\overrightarrow{c w}$, and note that $|\vec{p}|,|\vec{q}| \leq \omega$. We thus have

$$
\begin{aligned}
2|\operatorname{Area}(\triangle u v w)-\operatorname{Area}(\triangle a b c)| & \leq|\vec{p} \times \overrightarrow{a c}|+|\overrightarrow{a b} \times \vec{q}|+|\vec{p} \times \vec{q}| \\
& \leq \omega(|\overrightarrow{a b}|+|\overrightarrow{a c}|+\omega) .
\end{aligned}
$$




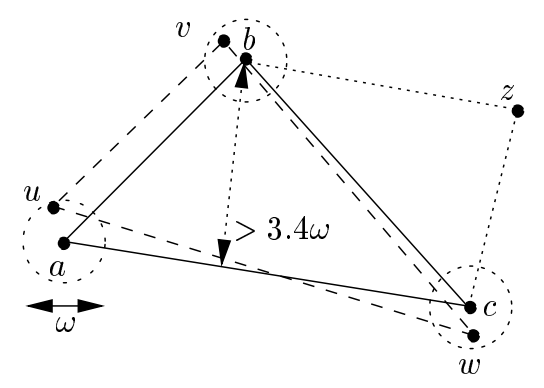

Fig. 3. Illustration to Lemma 3.5.

Let $h_{a b}, h_{a c}$ denote the heights of $\triangle a b c$ to the sides $a b, a c$, respectively. By assumption, we have

$$
h_{a b}, h_{a c},|a b|,|a c| \geq 3.4 \omega
$$

which implies that

$$
\begin{aligned}
2|\operatorname{Area}(\triangle u v w)-\operatorname{Area}(\triangle a b c)| & \leq \frac{5}{17}\left(|a b| \cdot h_{a b}+|a c| \cdot h_{a c}+\frac{5}{17}|a b| \cdot h_{a b}\right) \\
& =\frac{390}{289} \operatorname{Area}(\triangle a b c)
\end{aligned}
$$

or

$$
\operatorname{Area}(\triangle u v w) \geq\left(1-\frac{195}{289}\right) \operatorname{Area}(\triangle a b c)=\frac{94}{289} \operatorname{Area}(\triangle a b c) .
$$

This establishes (a).

To prove (b), we note that $\operatorname{Area}(\Delta v w z)$ is maximized when $z$ is a vertex of the region $\Delta(\tau)$. Using the fact that the slope of $u v$ is almost the same as that of $b c$, it can be shown that the point $z$ maximizing $\operatorname{Area}(\triangle v w z)$ must coincide with an endpoint of the edge of $\Delta(\tau)$ parallel to $b c$ and lying on the opposite side of $a$; see Fig. 2(i). In this case $d\left(z, \ell_{b c}\right)=\tau d\left(a, \ell_{b c}\right)$ and $\operatorname{Area}(\triangle b c z)=\tau \operatorname{Area}(\triangle a b c)$.

Arguing as in (a), we have

$$
2 \operatorname{Area}(\triangle b c z)=|\overrightarrow{z b} \times \overrightarrow{z c}|
$$

and

$$
2 \operatorname{Area}(\Delta v w z)=|\overrightarrow{z v} \times \overrightarrow{z w}|=|(\overrightarrow{z b}+\overrightarrow{b v}) \times(\overrightarrow{z c}+\vec{c} \vec{w})|
$$

Note that $|\overrightarrow{b v}|,|\overrightarrow{c w}| \leq \omega / 2$. We thus have

$$
\begin{aligned}
2|\operatorname{Area}(\triangle v w z)-\operatorname{Area}(\triangle b c z)| & \leq|\overrightarrow{b v} \times \overrightarrow{z c}|+|\overrightarrow{z b} \times \overrightarrow{c w}|+|\overrightarrow{b v} \times \overrightarrow{c w}| \\
& \leq \frac{\omega}{2}\left(|\overrightarrow{z b}|+|\overrightarrow{z c}|+\frac{\omega}{2}\right) .
\end{aligned}
$$

The two vertices $z_{1}, z_{2}$ of $\Delta(\tau)$ where $z$ can lie satisfy $\vec{b}_{1}=\tau \overrightarrow{a c}$ and $c \vec{z}_{2}=\tau \overrightarrow{a b}$. Consider the vertex $z_{1}$ (the treatment of $z_{2}$ is fully symmetric). We have

$$
\left|\overrightarrow{b z}_{1}\right|=\tau|\overrightarrow{a c}|
$$


and

$$
\left|\vec{c} \vec{z}_{1}\right|=\left|\overrightarrow{c b}+\overrightarrow{b z_{1}}\right|=|\overrightarrow{c b}+\tau \overrightarrow{a c}|=|(\tau-1) \overrightarrow{a c}+\overrightarrow{a b}| \leq(\tau-1)|\overrightarrow{a c}|+|\overrightarrow{a b}| .
$$

Hence

$$
\frac{\omega}{2}\left(\left|\overrightarrow{z_{1}} b\right|+\left|\overrightarrow{z_{1}} c\right|+\frac{\omega}{2}\right) \leq \frac{\omega}{2}\left((2 \tau-1)|\overrightarrow{a c}|+|\overrightarrow{a b}|+\frac{\omega}{2}\right) .
$$

Using the inequalities (3.8), we obtain, as in (a),

$$
2|\operatorname{Area}(\triangle v w z)-\operatorname{Area}(\triangle b c z)| \leq\left(\frac{5(2 \tau-1)}{17}+\frac{5}{17}+\frac{25}{578}\right) \operatorname{Area}(\triangle a b c),
$$

or

$$
\operatorname{Area}(\triangle v w z) \leq \operatorname{Area}(\triangle a b c)\left(\tau+\frac{5 \tau}{17}+\frac{25}{1156}\right)
$$

as asserted, thus establishing (b).

Finally, to prove (c), suppose that the width of $\triangle a b c$ is the height $h_{b c}$ to the edge $b c$. Then $\triangle a b c$ is contained in the strip $\sigma$ of width $h_{b c}$ whose boundary lines pass through the edge $b c$ and the vertex $a$. The strip of width $h_{b c}+\omega$, obtained by translating each line of $\sigma$ by $\omega / 2$ away from $\sigma$, contains $u, v$, and $w$. Therefore,

$$
\operatorname{Width}(\triangle u v w) \leq \operatorname{Width}(\triangle a b c)+\omega .
$$

The reverse inequality is proved in exactly the same manner.

The first two parts of the above lemma along with the fact that $\tau \geq 1$ imply the following.

Corollary 3.6. $\operatorname{Area}(\Delta v w z)<4.05 \tau \cdot \operatorname{Area}(\triangle u v w)$.

We are now in position to prove the main result of this section.

Theorem 3.7. Suppose there exists $\omega>0$ such that for each $p \in S^{*}$ there exists an annulus of width $\omega$ that encloses $A^{*} \cup\{p\}$. Then there exists an annulus of width at most $55.6 t \omega$ that encloses $S^{*}$.

Proof. If Width $\left(A^{*}\right) \leq 6.95 \omega$, then Corollary 3.2(ii) implies that the width of $S^{*}$ is at most $6.95(4 t-1) \omega$. Since a slab can be regarded as a degenerate annulus, $S^{*}$ can be enclosed by an annulus of width at most $55.6 t \omega$. So assume that $\operatorname{Width}\left(A^{*}\right) \geq 6.95 \omega$.

Suppose, without loss of generality, that $\triangle a_{1}^{*} a_{2}^{*} a_{3}^{*}$ is the largest-area triangle spanned by three of the points of $A^{*}$. We have

$$
\operatorname{Width}\left(\triangle a_{1}^{*} a_{2}^{*} a_{3}^{*}\right) \geq \operatorname{Width}\left(A^{*}\right) / 2>3.4 \omega .
$$

Fix a point $q \in S^{*}$. By Corollary 3.2(ii), the area of each of the triangles $\triangle a_{1}^{*} a_{2}^{*} q$, $\triangle a_{1}^{*} a_{3}^{*} q, \triangle a_{2}^{*} a_{3}^{*} q$ is at most $(4 t-1) \cdot \operatorname{Area}\left(\triangle a_{1}^{*} a_{2}^{*} a_{3}^{*}\right)$. Let $\mathcal{A}$ be an annulus of width $\omega$ that contains $A^{*} \cup\{q\}$, and let $C$ be the mid-circle of $\mathcal{A}$. Let $\mathcal{A}^{*}$ be the annulus of width 
55.6t $\omega$ that has $C$ as its mid-circle. We claim that $\mathcal{A}^{*}$ contains $S^{*}$. Indeed, let $q^{\prime}$ be any point of $S^{*}$, and let $\mathcal{A}^{\prime}$ be an annulus of width $\omega$ that contains $A^{*} \cup\left\{q^{\prime}\right\}$. Let $C^{\prime}$ be the mid-circle of $\mathcal{A}^{\prime}$. Clearly, $C, C^{\prime}$, and $\triangle a_{1}^{*} a_{2}^{*} a_{3}^{*}$ satisfy the conditions in Lemma 3.3 (with $\tau=4 t-1)$, which implies

$$
d(q, C) \leq(6.95(4 t-1)+3.5) \omega \leq 27.8 t \omega,
$$

implying that $q \in \mathcal{A}^{*}$, as claimed.

\section{Approximating $\Sigma^{*}(S)$}

In this section we apply the results of the preceding section to obtain an algorithm for computing a cylindrical shell of width at most $O\left(\omega^{*}(S)\right)$ that encloses an $n$-element point set $S \subset \mathbb{R}^{3}$. We first describe an algorithm for computing a subset $A \subseteq S$ of four points so that $\mu(A) \geq(1-\varepsilon) \mu(S)$, for some constant $\varepsilon>0$; recall that $\mu(X)$ is the maximum volume of a simplex spanned by the points of $X$.

Lemma 4.1. Given a set of $n$ points in $\mathbb{R}^{3}$ and a parameter $\varepsilon>0$, we can compute in $O\left(n \log (1 / \varepsilon)+(1 / \varepsilon)^{4.5} \log (1 / \varepsilon)\right)$ time a subset $A$ of four points so that $\mu(A) \geq$ $(1-\varepsilon) \mu(S)$.

Proof (Sketch). We first compute a box $B$ enclosing $S$ whose volume is at most $1+$ $\varepsilon$ times the minimum volume of any box containing $S$. This can be done in $O(n+$ $1 / \varepsilon^{4.5}$ ) time using the algorithm of Barequet and Har-Peled [7]. Suppose, with no loss of generality, that $B$ is axis-aligned and the coordinates of the endpoints of its main diagonal are $(0,0,0)$ and $\left(l_{x}, l_{y}, l_{z}\right)$. Choose a sufficiently large constant $c>1$ and set $\alpha=\varepsilon / c$. Draw a three-dimensional grid

$$
\left\{\left[i \alpha l_{x},(i+1) \alpha l_{x}\right] \times\left[j \alpha l_{y},(j+1) \alpha l_{y}\right] \times\left[k \alpha l_{z},(k+1) \alpha l_{z}\right] \mid 0 \leq i, j, k \leq\lceil 1 / \alpha\rceil\right\}
$$

of size $O\left(1 / \alpha^{3}\right)$. Let $Q$ be the set of grid vertices adjacent to the grid cells that contain at least one point of $S$. $Q$ can be computed in $O\left(n \log (1 / \varepsilon)+1 / \varepsilon^{3}\right)$ time. For each pair $1 \leq i, j \leq\lceil 1 / \alpha\rceil$, if there are more than two points in $Q$ whose $x$ - and $y$-coordinates are $i$ and $j$, respectively, we keep only two of them-the ones with the maximum and minimum values of $k$. $Q$ now has at most $O\left(1 / \alpha^{2}\right)$ points. We then compute, in $O\left(\left(1 / \alpha^{2}\right) \log (1 / \alpha)\right)$ time, the set $V \subseteq Q$ of vertices of the convex hull of $Q$. By a result of Andrews [6], $|V|=O\left(1 / \alpha^{3 / 2}\right)$. Next, we compute in $O\left(|V|^{3} \log |V|\right)$ time the largest volume tetrahedron $q_{1} q_{2} q_{3} q_{4}$ spanned by $V$ (we omit details of the rather straightforward algorithm for doing so). Let $a_{i} \in S$ be a nearest neighbor of $q_{i}$, for $i=1, \ldots, 4$. We return $A=\left\{a_{1}, a_{2}, a_{3}, a_{4}\right\}$. Using a somewhat tedious analysis, similar to the one in [7], it can be shown that $\mu(A) \geq(1-\varepsilon) \mu(S)$.

Set $\varepsilon=\frac{1}{140}$ and compute in $O(n)$ time a set $A \subseteq S$ of four points such that $\mu(A) \geq$ $(1-\varepsilon) \mu(S)$, using the above lemma. Let $\mathbb{S}^{2}$ denote the unit sphere of directions in $\mathbb{R}^{3}$. For each $q \in S$ we define a real-valued function $F_{q}$ on $\mathbb{S}^{2}$, so that, for $\mathbf{n} \in \mathbb{S}^{2}, F_{q}(\mathbf{n})$ is the width of a thinnest annulus within the plane $\pi^{(\mathbf{n})}$ that contains the orthogonal 
projections of $A \cup\{q\}$ on the plane $\pi^{(\mathbf{n})}$. Clearly, $F_{q}$ is a piecewise-algebraic function of "constant description complexity" (in the terminology of [16]). Let $E$ denote the pointwise maximum of $\left\{F_{q}\right\}_{q \in S}$, let $\mathbf{n} \in \mathbb{S}^{2}$ be a direction that minimizes $E$, and let $\omega=E(\mathbf{n})$.

Lemma 4.2. $\omega \leq \omega^{*}(S) \leq 56 \omega$.

Proof. The fact that $\omega=\min _{\mathbf{v} \in \mathbb{S}^{2}} \max _{q \in S} F_{q}(\mathbf{v})$ implies that, for each $\mathbf{v} \in \mathbb{S}^{2}$, there exists $q \in S$ such that any cylindrical shell that contains $A \cup\{q\}$ and has axis-direction $\mathbf{v}$ must have width at least $\omega$. Hence the minimum width of a cylindrical shell that encloses $S$ is at least $\omega$.

On the other hand, since $\mu(A) \geq(1-\varepsilon) \mu(S)$, which corresponds to setting $t=$ $1 /(1-\varepsilon)=7 / 6.95$ in Lemma 3.3, Theorem 3.7 implies that there exists a cylindrical shell with axis-direction $\mathbf{n}$ and width at most $55.6 \cdot t \omega=56 \omega$ that contains $S$.

The algorithm is now straightforward. We compute $E$ in $O\left(n^{2+\delta}\right)$ time, for any $\delta>0$, using, e.g., the algorithm of [4], and then examine each vertex, edge, and face of (the graph of) $E$ to find the global minimum of $E$. Suppose the minimum is attained at some direction $\mathbf{n}$ by a point $q \in S$. We project $S$ orthogonally onto $\pi^{(\mathbf{n})}$, and compute the minimum-width annulus $\mathcal{A}$ within $\pi^{(\mathbf{n})}$ that contains the projected set $S^{*}$. This can be done in additional time $O\left(n^{2}\right)$ [12]. (Alternatively, we can compute in $O(1)$ time the radius $\rho$ and the mid-circle $C^{*}$ of the minimum width annulus containing $A^{(\mathbf{n})} \cup\left\{q^{(\mathbf{n})}\right\}$ and set $\mathcal{A}$ to be the annulus of width $56 \rho$ and with mid-circle $C^{*}$.) We then "lift" $\mathcal{A}$ in the direction $\mathbf{n}$ to obtain a cylindrical shell, of the same width, that encloses $S$. By the preceding analysis, we obtain the following.

Theorem 4.3. Given a set $S$ of $n$ points in $\mathbb{R}^{3}$, one can compute, in $O\left(n^{2+\delta}\right)$ time, for any $\delta>0$, a cylindrical shell that contains $S$, whose width is at most $56 \omega^{*}(S)$.

Remark 4.4. We believe that our approach can be strengthened to give a near-lineartime algorithm. Intuitively, we need to show that one does not have to search over all directions $\mathbf{n} \in \mathbb{S}^{2}$. Instead, we conjecture that it suffices to search over the onedimensional locus of axis directions of cylinders that pass through four points of $S$ that span a "large-volume" simplex. However, at present we do not know whether this holds.

\section{Conclusions}

In this paper we presented a constant-factor approximation algorithm for the minimumwidth cylindrical shell problem that runs in near-quadratic time. We also presented an algorithm for computing the thinnest cylindrical shell containing a point set. We conclude by mentioning two open problems:

1. Is there a faster algorithm for computing the minimum-width cylindrical shell containing a point set in $\mathbb{R}^{3}$ ?

2. Develop a $(1+\varepsilon)$-approximation algorithm for the minimum-width cylindrical shell problem that runs in near-linear time (or even in near-quadratic time). 


\section{Acknowledgments}

The authors thank Sariel Har-Peled for many useful discussions and two anonymous referees for their helpful comments.

\section{References}

1. P. K. Agarwal, B. Aronov, and M. Sharir, Line transversals of balls and smallest enclosing cylinders in three dimensions, Discrete Comput. Geom. 21 (1999), 373-388.

2. P. K. Agarwal, B. Aronov, S. Har-Peled, and M. Sharir, Approximation and exact algorithms for minimumwidth annuli and shells, Discrete Comput. Geom. 24 (2000), 687-705.

3. P. K. Agarwal and J. Matoušek, On range searching with semialgebraic sets, Discrete Comput. Geom. 11 (1994), 393-418.

4. P. K. Agarwal, O. Schwarzkopf, and M. Sharir, The overlay of lower envelopes in 3-space and its applications, Discrete Comput. Geom. 15 (1996), 1-13.

5. P. K. Agarwal and M. Sharir, Efficient randomized algorithms for some geometric optimization problems, Discrete Comput. Geom. 16 (1996), 317-337.

6. G. E. Andrews, A lower bound for the volumes of strictly convex bodies with many boundary points, Trans. Amer. Math. Soc. 106 (1965), 270-273.

7. G. Barequet and S. Har-Peled, Efficiently approximating the minimum-volume bounding box of a point set in three dimensions, Proc. 10th Annu. ACM-SIAM Sympos. Discrete Algorithms, 1999, pp. 82-91.

8. M. de Berg, M. van Kreveld, M. Overmars, and O. Schwarzkopf, Computational Geometry: Algorithms and Applications, Springer-Verlag, Berlin, 1997.

9. T. M. Chan, Approximating the diameter, width, smallest enclosing cylinder, and minimum-width annulus, Proc. 16th ACM Sympos. Comput. Geom., 2000, pp. 300-309.

10. O. Devillers and F. P. Preparata, Evaluating the cylindricity of a nominally cylindrical point set, Proc. 11th ACM-SIAM Sympos. Discrete Algorithms, 2000, pp. 518-527.

11. C. A. Duncan, M. T. Goodrich, and E. A. Ramos, Efficient approximation and optimization algorithms for computational metrology, Proc. 8th ACM-SIAM Sympos. Discrete Algorithms, 1997, pp. 121-130.

12. H. Ebara, N. Fukuyama, H. Nakano, and Y. Nakanishi, Roundness algorithms using the Voronoi diagrams, Abstracts 1st Canad. Conf. Comput. Geom., 1989, p. 41.

13. L. W. Foster, GEO-METRICS II: The Application of Geometric Tolerancing Techniques, Addison-Wesley, Reading, MA, 1982.

14. J. García-Lopez and P. Ramos, Fitting a set of points by a circle, Proc. 13th Annu. ACM Sympos. Comput. Geom., 1997, pp. 139-146.

15. E. Schömer, J. Sellen, M. Teichmann, and C. Yap, Efficient algorithms for the smallest enclosing cylinder problem, Algorithmica 27 (2000), 170-186.

16. M. Sharir and P. K. Agarwal, Davenport-Schinzel Sequences and Their Geometric Applications, Cambridge University Press, Cambridge, 1995.

17. K. Suresh and H. B. Voelcker, New challenges in dimensional metrology: A case study based on "size," Manufacturing Rev. 7 (1994), 292-303.

18. R. K. Walker and V. Srinivasan, Creation and evolution of the ASME Y14.5.1M standard, Manufacturing Rev. 7 (1994), 16-23.

Received May 31, 2000, and in revised form October 25, 2000. Online publication August 29, 2001.

Note added in proof. Recently Har-Peled and Varadarajan have developed a $(1+\varepsilon)$ approximation algorithm for the minimum-width cylindrical shell problem whose running time is $n / \varepsilon^{O(1)}$. 